When all had been removed there remained a smooth-walled cavity the size of a small walnut. Everywhere it seemed bounded by thin bony parietes, except superiorly, where there was a distinct sensation of yielding, as though the dura mater were exposed. The lateral sinus could not be seen, being separated from the cavity by a thin lamina. Anteriorly the cavity freely communicated with the auditory canal and tympanum by several large round openings. The cavity was plugged with iodoform gauze and the flap was readjusted. The unhealthy soft parts corresponding to the sinus in the base of the flap were cut away, leaving a large opening into the cavity. Under the microscope a small flake of the substance showed fat cells, a large quantity of cholesterine and amorphous débris with large flattened cells like those of squamous epithelium. There was a total absence of signs of caries, no fragments of bone or gritty particles being detected.

March 14th. - The cavity has been washed out with " red wash" and plugged daily. It is filling up with healthy granulations, and epithelium is advancing over it with remarkable rapidity from the opening into the auditory canal. 'The general health is excellent.

March 26th. - The cavity is now quite dry and the granulations seen through the speculum have disappeared. There is a certain amount of hearing to loud conversation, but the facial paralysis remains. The engraving, from a drawing by Mr. Burgess, gives a good idea of the extent of the cavity. The curved probe can be passed into it from the bony auditory canal and out of the external aperture.

April 6th.-The opening was closed by a simple plastic operation.

May 2nd.-The patient went to a convalescent home. The opening behind the ear was completely covered in. All discharge had ceased from the canal. The hearing for conversation was good. 'The girl's health and intelligence have markedly improved. She is cheerful and assists in the ward work, and her apathetic condition observed on admission has quite disappeared, the change in the mental condition seeming, indeed, the most striking peculiarity about the case.

Stratford-place, $w$.

\section{ARTHRODESIS, OF THE ANKLE-JOINT IN TALIPES VARUS DUE TO INFANTILE} PARALYSIS.

BY EDWARD COTTERELL, F.R.C.S. ENG., SURGEON TO THE WEST-END HOSPITAL FOR PARALYSIS AND DISEASES OF THE NERVOUS SYSTEM AND SURGEON (OUT-PATIENTS) AT THE LONDON LOCK HOSPITAL ETC.

ARTHRODESIS, or the establishment of ankylosis in a healthy joint, with the idea of converting a yielding and flail-like limb into one which will bear the weight of the body and be useful for locomotion, is an operation which has lately attracted the attention of surgeons both in this country and abroad. Within the last twelve months my colleague, Dr. Wallis Ord, has kindly placed three children suffering from paralytic talipes varus under my charge at the West-end Hospital for Paralysis. Of these cases the knee was only affected in one patient and in that one not badly enough to warrant resection of the joint. When the first case came under my care I thought excision of the ankle-joint a rather severe measure, as it would necessarily retard the growth of the limb from grave interference with the lower epiphyses of the tibia and fibula. On considering the matter I came to the conclusion that, as the joint in these cases is not diseased, it would be possible to cause an ankylosis without removing the amount of bone, such as is generally done in an ordinary resection. The operation, which I have devised and have now practised in three cases, is as follows: An anterior incision is made over the joint from one malleolus to the other, the dorsalis pedis artery being divided and both ends tied. The tendons of the extensor proprius hallucis and tibialis anticus, as also those of the extensor communis digitorum, are divided, sutures being previously passed above and below the line of incision, to facilitate their being reunited when the wound is closed. The anterior tibial nerve is treated in the same way. The joint is then well opened and the astragalus dislocated forwards. The whole of the articular cartilage of the upper surface of the astragalus is now removed with a probe-pointed knife, as well as the cartilage from the inner and outer facets, where the malleoli articulate. The lower articular surfaces of the tibia and fibula are treated in the same manner, the articular cartilage being removed from the two malleoli. No particular care is taken to cut a smooth surface; in point of fact the pseudoarticular surfaces are purposely left rough. Having thus cleared away the whole of the articular cartilage, the cut tendons are united, the nerve sutured, and the skin wound brought together secundum artem. The limb is put up on a splint for three or four days in an everted position, after which time it is treated with a plaster of Paris splint for three months. Even after this time it is wise, in the case of children, to have a well-fitting boot made with side supports, which should be worn for another twelve months, or until one may fairly assume that firm bony union bas occurred. I claim the following advantages for this method of resection of the ankle-joint: (1) It interferes very little with the growth of the leg bones, as the epiphyses are not removed; (2) by leaving the malleoli, good lateral support is maintained, a very essential thing in the class of cases for which the operation is recommended; (3) the operation is easy to perform and is not at all dangerous ; (4) the result, as regards power of locomotion afterwards, is very good; (5) expensive and cumbersome apparatus can be sub. sequently dispensed with.

West Halkin-street, Belgrave-square, S.W.

\section{A CASE OF NOCTURNAL SPASM OF THE LARYNX IN AN ADULT.}

BY GEORGE HERSCHELL, M.D. LOND,

PHYSICIAN TO THE NAIIONAL HOSPITAL FOR DISEASES OF THE HEART ETC.

CASES of this nature are of such extreme rarity that I feed I have an excuse for briefly recording one which I have had close opportunities of observing during the last few months. The patient whose case I am about to describe is a literary man thirty-six years of age of a markedly neurasthenic temperament. About ten years ago he suffered very severely for some months from dyspepsia, but recovered, and now he enjoys very good health in this respect. This dyspepsia was of the atonic form and originated in an exhausted condition of the nervous system brought on by overwork and worry. On physical examination he appears perfectly normal, the pupils and radial pulses being equal, the heart normal, and there being no abnormal pulsations, bruits, thrills, or dulness in the thorax. The patellar reflexes are normal, and there is no incoördination. He first noticed spasm of the larynx at night about eighteen months ago and at that period used to have at least two attacks a week. On more than one occasion two attacks have occurred in the same night. This state of things continued for two months, after which under appropriate treatment the attacks lessened in frequency and finally ceased. He was then free for several months. Having relaxed his hygienic measures he had another attack on March 30th, 1893, and again one on April 14th. This last one was the most severe that he had ever experienced and in it he very nearly lost consciousness. A typical attack occurs in the following manner: Having retired to bed in his usual health after having dined a little later than customary and over-indulged somewhat in the matter of cigars, he wakes at about 2 o'clock with a sense of suffocation due to a spasm of the larynx, which completely or almost completely prevents inspiration. This continues for a few seconds, when the spasm is partial the air being very slowly drawn into the larynx with a crowing sound. If the spasm be complete at first, it always partially relaxes after a few seconds and allows the air to slowly enter the lungs in the manner described. After nearly a minute the spasm entirely relaxes, but the larynx feels very sore and uncomfortable for an hour or two. During the attack the heart palpitates violently, and on examination by percussion directly afterwards the stomach is found to be tympanitic and evidently much distended. If the patient can bring up the flatus he is comfortable, goes to sleep at once, and has no more attacks that night. Very often, however, there appears to be a spasmodic condition of the orifices of the stomach which prevents the passage of flatus either npwards or downwards. Under these circumstances the patient is afraid to 
go to sleep, but probably does so from sheer exhaustion and most likely has another attack before the morning. As a rule he has been lying on his back when an attack has taken place

Spasm of the larynx or laryngismus stridulas is of very rare occurrence in the adult, and the text-books of medicine have very little to say about it. Neither Fagge nor Osler mentions it at all, and Taylor simply says : "This occurs most frequently in connexion with laryngitis, cedema of the larynx, paralytic conditions, or the presence of foreign bodies; it may also be a danger in epilepsy, tetanus, hydrophobia, or chorea. The entrance of saliva or of small particles of food or drink into the larynx may cause most dangerous spasm, and a certain amount is often induced by the application of medicated solutions to the mucous membrane of the larynx. Apart from these causes it is most of ten the result of hysteria. Bosworth, ${ }^{1}$ however, a recent American writer, gives a very good account of the condition and reports several cases due to reflex irritation proceeding respectively from deflected septum, hypertrophic rhinitis, nasal polypus and chronic pharyngitis. The conclusions at which he arrives are as follows: It does not ordinarily involve any danger to life. In most instances it is purely reflex. There must be a somewhat abnormal irritability of the nervous system as a predisposing cause. It may be excited by the entrance of food or other foreign substances into the larynx. It is usually reflex to some diseased condition of the larynx or other portion of the upper air tract. He considers that the irritation generally proceeds from the larynx itself and that the mouth breathing caused by the nasal stenosis produces sufficient irritation and dryness of the laryngeal mucous membrane to start the reflex impulse which initiates the laryngeal spasm. Bosworth does not mention reflex irritation proceeding from the stomach as a possible cause, and I am unable to find it given as such in any medical work with which I am acquainted. My case therefore has a certain interest insomuch as the attacks were certainly excited by the presence of undigested food in the stomach, although the tobacco smoking probably predisposed to them by rendering the larynx more irritable. In forming an opinion in a case of this kind there are several possibilities to be borne in mind : (1) That it might be of an epileptiform nature; (2) that it might be a laryngeal crisis in commencing tabes; (3) that it might be due to pressure from an aneurysm (4) that it might be simply caused by the entrance of saliva into the larynx. At one time I was under the impression that the last might have been the cause, but as the attacks occurred when the patient's head was placed in such a posi tion that any saliva which was secreted ran out on to the pillow, and as on one occasion, to make sure, he slept all night with a dentist's saliva ejector in his mouth and yet had two attacks that night, I was forced to abandon the hypothesis. The time that elapsed and the improvement under treatmen negatived the idea of aneurysm. In the laryngeal crises of tabes there are usually other signs present, such as loss of tendon reflex and abnormalities of the pupils, which would lead to a correct opinion being formed of the case.

Queen Anne-street, W.

\section{THE TREATMENT OF MYXEDEMA AND CRETINISM.}

By G. R. MURRAY, B.A., M.B. Cantab , M.R.C.P. Lond, LECTURER ON BACTERIOLOGY IN THE UNIVERSITY OF DURHAM COLLEGE OF MEDICINE; PATHOLOSIS T AT THE HOSPITAL FOR SICK CHILDREN, NEWCASTLE-ON-TYNE.

A LARGE number of cases of nyxoedema and several sporadic cretins are being treated at the present time by thyroid juice in one form or another, so that a few observations which have been made during the last two years in the course of treating eight cases of myxodema may be of interest. So many successful cases have now been recorded that it is quite evident that in thyroid juice there exists a remedy for nyxœedema and allied conditions. The normal thyroid gland supplies an "internal secretion" which is necessary to maintain the body in health. When, owing to disease or removal of the gland, a sufficient amount of this secretion is no longer formed a condition supervenes now well known as myxcedema, cretinism, or cachexia strumipriva. The intensity of the symptoms depends upon the

\section{amount of secreting tissue which is affected. In cases} where the symptoms are only slight, part of the gland or an accessory gland is still supplying a small but insufficient quantity of the secretion. It has now been clearly shown that the same secretion obtained from the thyroid gland of some healthy animal, such as a sheep, a pig, or a cow, when injected beneath the skin or given by the mouth, can carry on the functions which were performed by the normal thyroid secretion before the gland was affected. One can thus remove the symptoms of these conditions by supplying what is wanting. In a similar manner one can supplement deficiencies in gastric, pancreatic or other glandular secretions by preparations of the stomach, pancreas or other gland of one of the lower animals. If one bears these facts in mind it is easy to adapt the treatment to any given case, and provided that no incurable degeneration has taken place one can restore the patient to a condition of health and prevent the gradual degeneration of the nervous and vascular systems which so frequently takes place in the later stages of myxœdema. The remedy has been given in various forms. For hypodermic injection I have always employed a glycerine extract of sheep's thyroid gland. This extract is composed of equal parts of thyroid juice, glycerine and a $\frac{1}{2}$ per cent. solution of carbolic acid. A drachm and a half of this extract is the product of one whole gland. It can be injected in doses of from five to fifteen minims ; larger doses may cause local irritation Since Dr. E. L. Fox and Dr. H. Mackenzie showed that thyroid juice could produce the same physiological effects when swallowed I have employed an extract of the same strength as the above, but without carbolic acid, for administration by the mouth. This can be prescribed in doses of from fire minims to twenty minims in water and has scarcely any taste. Both these preparations have been made by Messrs. Brady and Martin each week, and as they are made in considerable quantities the strength is practically constant. This is important, as it enables one to give doses of uniform strength of a preparation which does not disturb digestion. Raw and lightly-cooked thyroid glands have been given with good results in a considerable number of cases, but there are some disadvantages in giving the remedy in this form. The glands vary considerably in size, so that a fraction of a gland is not pleasant to take ; it is apt to set up gastrointestinal disturbances and its administration has twice had a fatal result. A glycerine extract contains the active material which is required for the treatment, so that the rest of the gland is not a unform dose. The raw gland is not required and possibly may be harmful. Mr. Hurry Fenwick has employed thyroid juice expressed from the fresh gland mixed with water for hypodermic injection. Dr. Arthur Davies has used a powder which has been prepared by Mr. White. Dr. Vermehren has given the white precipitate which is formed by adding alcohol and thyroid extract. Each of these different preparations has been found to be capable of remoring the symptoms of myxodema.

The treatment may conveniently be divided into two stages. During the first stage the object is to remove the symptoms of the disease. In the second one has to maintain the patient in the condition of health which has already been reached. In order to prevent disappointment it is important to explain to the patient that the improved condition can only be kept up by a continued use of the remedy for the rest of life and that for the future some thyroid preparation must constitute part of the regular diet. The first stage of the treatment has to be carried out gradually and with care, for the alteration which takes place in the patient's condition is so great that in many cases it is not safe to attempt to bring it about rapidly. This caution must be exercised especially in cases which show signs of cardiac or vascular degeneration. In these very small doses should be given a first and the effect on the pulse carefully watched. Some patients have to be kept in the house or even in bed for a time so as to prevent any unaccustomed strain being put on the heart. Two of my patients, who had signs of cardiac degeneration before the treatment was applied, died of syncope after the symptoms of myxoedema had greatly improved, brought on by unusual exertion undertaken too soon, before the heart had been able to recover or had become accustomed to the altered conditions of the body. Such patients should only be allowed to take increased excercise by degrees. This stage generally requires from six to twelve weeks' treatment, according to the state of the patient. In six weeks this part of the treatment has been carried out by injecting the extract. It has been 